\title{
Intracranial hydatid cyst: imaging findings of a rare disease
}

\author{
Bedros Taslakian, ${ }^{1}$ Houssein Darwish ${ }^{2}$
}

'Department of Radiology, New York University Langone Medical Center, New York, New York, USA

${ }^{2}$ Department of Diagnostic Radiology, American University of Beirut Medical Center, Beirut, Lebanon

\section{Correspondence to}

Dr Bedros Taslakian, btaslakian@gmail.com

Accepted 2 August 2016

\section{SUMMARY}

Hydatid disease (echinococcosis) is a worldwide zoonosis produced by the larval stage of the Echinococcus tapeworm. The disease is endemic in many parts of the world, particularly in the Middle East, Australia, New Zealand, South America and central and south Europe. Intracranial hydatid disease is considered a rare disease and may be sometimes very difficult to diagnose based on the clinical and laboratory findings. Therefore, it is important to be aware of the condition and the imaging findings even in the non-endemic parts of the world. We report the case of a 12-year-old boy who presented with headache and vomiting for a few months. The mass was totally excised, with no postoperative complications. We present MR spectroscopy (MRS) findings in this operatively proven case of hydatid cyst of the brain. We discuss imaging findings, in particular the findings on MRS, which is rarely reported in the literature.

\section{BACKGROUND}

Echinococcosis is a widespread zoonotic disease which frequently involves the liver, lungs and, rarely, the brain.

The most common agent is Echinococcus granulosus, which causes unilocular hydatid cyst formation. ${ }^{1}$ Although most cysts develop in the liver, some disseminate to other sites; such as the brain. $^{23}$ The larval stage can involve the brain via the choroid plexus. ${ }^{2}{ }^{4} 5$ Cerebral hydatid cyst are usually localised within the watershed zone of the middle cerebral arteries, often in the parietal lobe. ${ }^{2} 67 \mathrm{~A}$ hydatid cyst consists of a hydatid sac (vesicle filled with fluid) with a thin, adventitial layer (neural tissue reaction) that may contain scolexes scattered in a limpid fluid. ${ }^{8} 9$ The incidence of intracranial hydatid disease is $1-2 \%$ of all reported cases. Imaging is essential for diagnosis since clinical and laboratory findings are nonspecific. MRI and spectroscopy in particular has an important role in establishing an accurate presurgical diagnosis and differentiating this rare entity from other intracranial cystic lesions. Imaging findings on MR spectroscopy are rarely reported in the literature. We present MR spectroscopy findings in this operatively proven case of hydatid cyst of the brain and provide review of the literature.

\section{CASE PRESENTATION}

A previously healthy 12-year-old boy presented with a 6-month history of episodic headache and recurrent projectile vomiting. He described occasional blurry vision, and denied any seizure, dizziness and fever. On examination, the patient's oral temperature was $37.5^{\circ} \mathrm{C}$, and the oxygen saturation was $99 \%$ on room air; he had a heart rate of $75 \mathrm{bpm}$, a blood pressure of $115 / 73 \mathrm{~mm} \mathrm{Hg}$ and a respiratory rate of $20 \mathrm{~min}$. Neurological examination showed normal muscle strength, sensitivity and reflexes with no cranial nerve deficit. The heart, lung and abdominal examination were normal. The patient had normal developmental milestones.

\section{INVESTIGATIONS}

White cell count, haemoglobin and electrolytes were all normal at the time of presentation. Contrast-enhanced MRI of the brain was performed showing a well-defined right parietal cystic lesion, with a thick surrounding capsule showing no enhancement after contrast administration (figure 1A-C). The lesion had cerebrospinal fluid (CSF) signal on all MR sequences and the diffusion images were normal. There was minimal surrounding vasogenic oedema with mild compression of the occipital horn of the right lateral ventricle (figure 1A). MR spectroscopy of the central cystic part of the lesion showed increased choline and a peak at $2.4 \mathrm{ppm}$ representing pyruvate and succinate. MR spectroscopy of the peripheral solid part of the lesion showed increased choline/ $\mathrm{N}$-acetyl aspartate (NAA) ratio and a large peak of lipids (figure 1D, E). The MRI findings were suggestive of a hydatid cyst. The indirect haemagglutination test was $1: 32$ (reference $<1: 32$ ). Complete blood count revealed no eosinophilia. Chest X-ray and abdominal ultrasound scan were performed to look for systemic hydatidosis, and showed no pathology.

\section{DIFFERENTIAL DIAGNOSIS}

Based on the clinical and radiological findings, the differential diagnosis includes hydatid cyst, and less likely glial cyst, glioneuronal tumour and abscess.

\section{TREATMENT}

The patient underwent a right parietal craniotomy. The lesion was strongly adherent to the dura (figure 2A) and ruptured while being dissected from the surrounding cerebral tissue (figure 2B), with drainage of a large amount of yellowish gelatinous fluid. A frozen section of the lesion, which was completely resected was suggestive of a glial tumour. The surgical cavity was then irrigated with hypertonic saline $3 \%$.

\section{OUTCOME AND FOLLOW-UP}

The patient had an uneventful postoperative period and was discharged home on albendazole and 
A

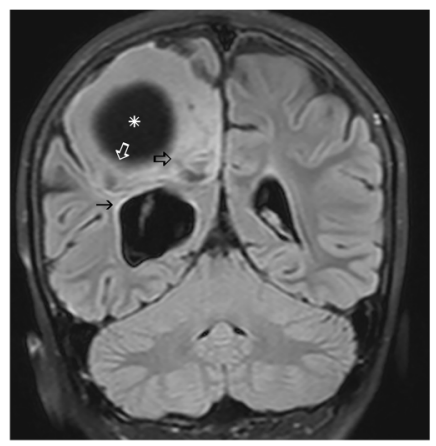

B

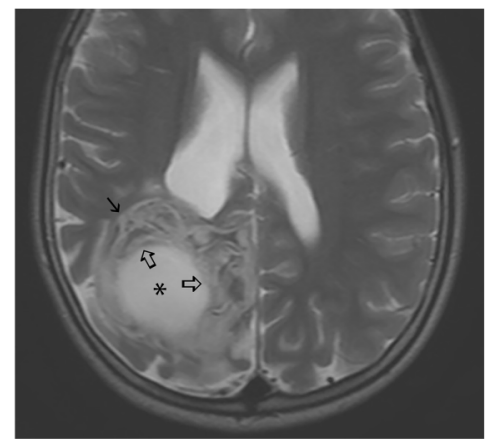

C

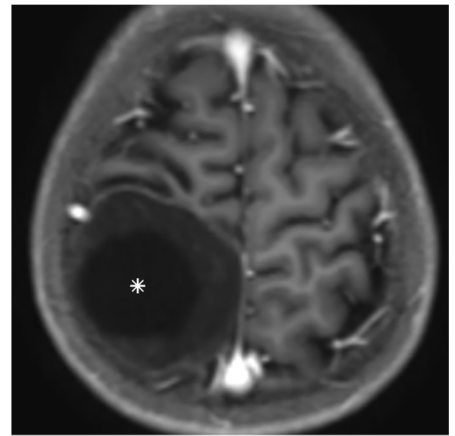

D

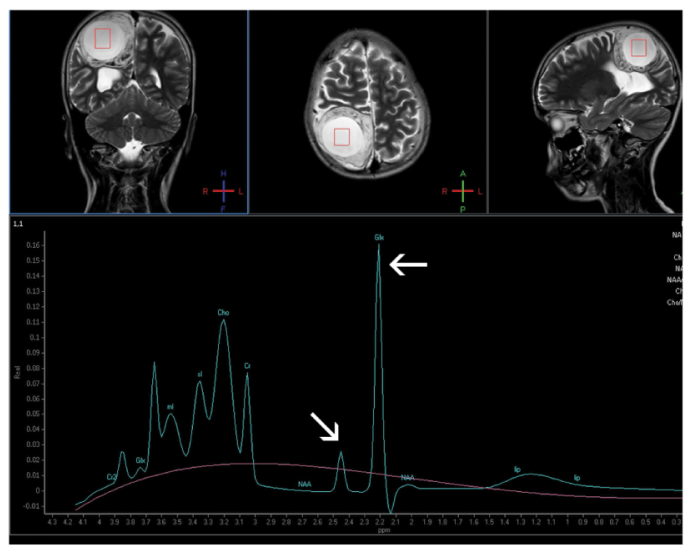

$\mathrm{E}$

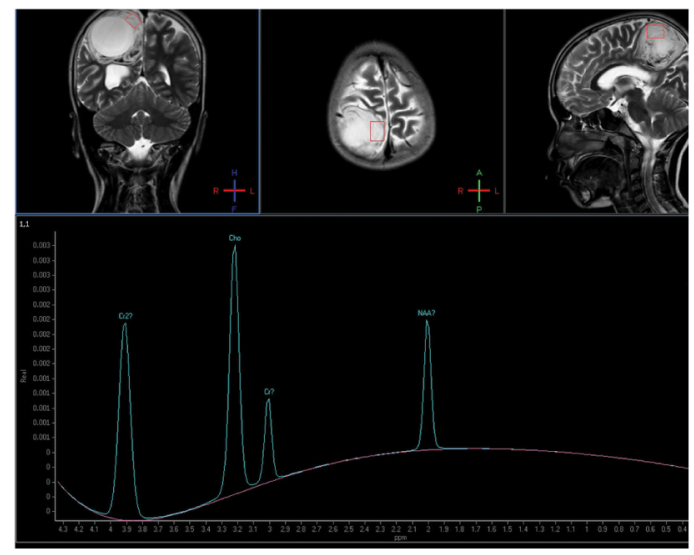

Figure 1 (A) Coronal fluid attenuation inversion recovery (FLAIR) MRI of the brain showing a large well-defined, unilocular, cerebrospinal fluid-like cyst ${ }^{*}$ ) located in the right parietal lobe. The cyst is causing minimal mass effect on the occipital horn of the right lateral ventricle (arrow) with mild surrounding vasogenic oedema. Collapsed membranes are seen in the medial and inferior aspect of the cyst (open arrows). (B) Axial T2-weighted MRI of the brain showing the right parietal hyperintense hydatid cyst $\left({ }^{*}\right.$ ). The cyst has a thin hypointense rim (arrow) with mild surrounding vasogenic oedema along its anterior aspect. Collapsed membranes are seen in its medial aspect with a ball-of-wool pattern (open arrows). (C) Axial T1-weighted MRI of the brain after gadolinium administration showing the right parietal hypointense hydatid cyst $\left({ }^{*}\right)$ with the non-enhancing collapsed membranes seen in its medial aspect. (D) Single voxel MR spectroscopy from the central cystic portion of the lesion showing increased choline, a small peak at 2.40-2.50 ppm representing pyruvate and succinate (oblique arrow), and a large abnormal peak at $2.2 \mathrm{ppm}$ representing glutamate/aminoacids (horizontal arrow). Pyruvate and succinate are well-known metabolic end products arising from microorganisms. (E) Single voxel MR spectroscopy from the peripheral solid portion of the cystic lesion showing increased choline/ $\mathrm{N}$-acetyl aspartate ratio.

Figure 2 (A) Intraoperative photograph showing the right parietal lobe lesion with its white wall. (B) Intraoperative photograph of the ruptured cystic lesion showing a large cavity of a unilocular cyst and its pearly white wall.
A

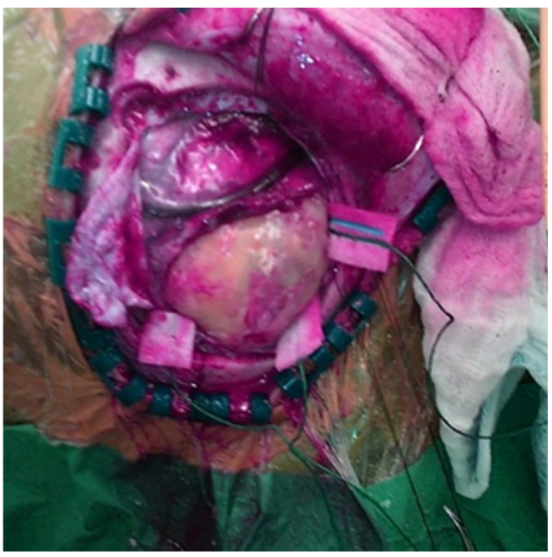

B

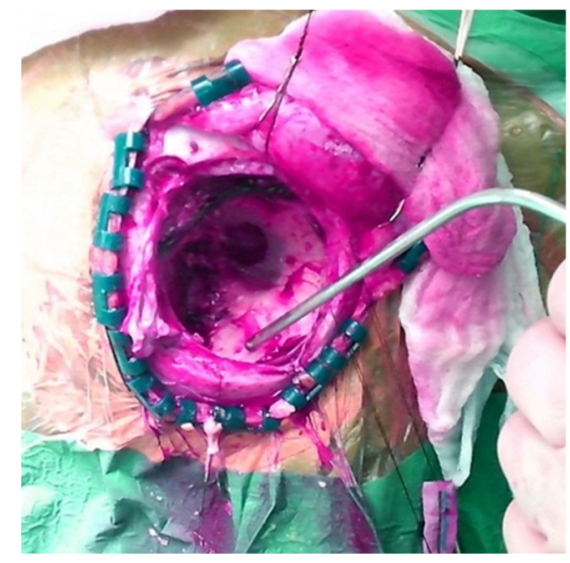

valproate sodium. Pathological examination of the surgical specimen was consistent with hydatid cyst with a thick collagenous laminated fibrotic cyst wall, inner germinal lining and a free scolex (figure 3A,B). Follow-up examinations showed no motor weakness or sensory deficit. Repeat ultrasound of the abdomen and pelvis and chest X-ray were all normal. Repeat MRI of the brain and spinal cord after 3 months showed the surgical cavity with no new lesions. 
Figure 3 (A) Low-magnification photomicrograph revealing lamellated fibrous and chitinous wall $\left(^{*}\right)$ and inner germinal lining (arrow). (B) High-magnification photomicrograph of the specimen demonstrating a thick collagenous laminated fibrotic cyst wall, the germinal membrane $\left(^{*}\right)$ with mononuclear reactive cells and a free scolex (open arrow) in the cyst lumen.
A

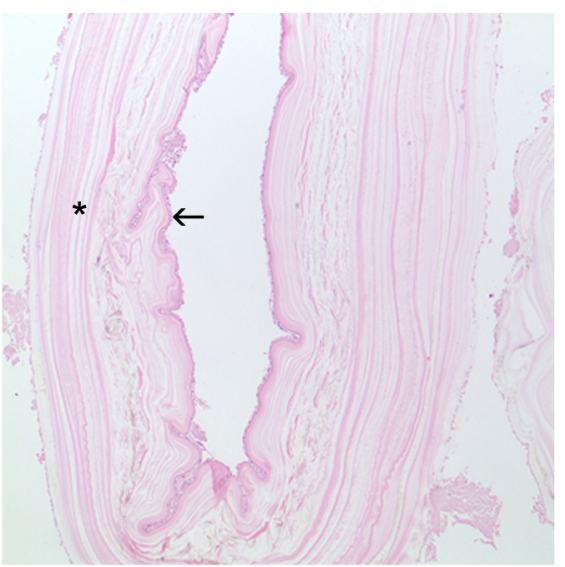

B

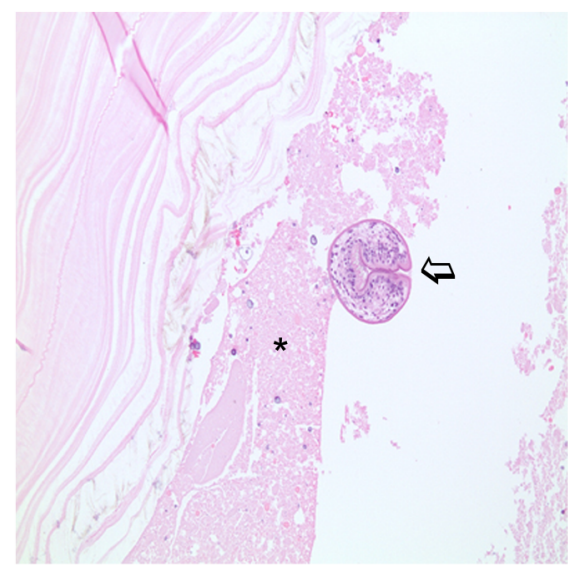

\section{DISCUSSION}

Hydatid disease is a worldwide zoonosis commonly seen in the Middle East, Mediterranean countries, South America, Africa, New Zealand and Australia. ${ }^{1}$ It is produced by the larval stage of the Echinococcus tapeworm, with the most frequently encountered type in humans being E. granulosus. ${ }^{1}$ Central nervous system involvement is very rare representing only $1 \%$ of all cases. ${ }^{1}$ In addition, intracranial hydatid disease represents $1-2 \%$ of all cerebral space-occupying lesions. ${ }^{2-4}$ Cerebral hydatid disease is usually diagnosed during childhood and is often solitary. ${ }^{1} 24^{5}$ Multiple cerebral hydatid cysts bring rare usually result from dissemination secondary to spontaneous, traumatic or surgical rupture of a primary cyst. ${ }^{6-8}$ Although hydatid disease may be located anywhere in the brain, it is most frequently found in both hemispheres, particularly in the middle cerebral artery territory with the parietal lobe being the most common site. ${ }^{134}$ Very rarely the cysts are located in the posterior cranial fossa or ventricles. ${ }^{3} 9$ In patients with cerebral hydatid disease, concurrent hepatic, pulmonary and other organ involvement has been reported. ${ }^{3}{ }^{10-12}$ In our case, no other pathology was detected outside the brain.

Cerebral hydatid cysts may not show any symptoms until they reach a considerably large size. They usually present with focal neurological deficit and features of raised intracranial pressure; the latter results from the large size or interference with CSF flow. Most patients present with headache and vomiting, followed by motor weakness and seizures. In addition, it is worth mentioning that the antibody response in cases of intracranial hydatid disease, as also noted in our case, is usually absent or very low, especially when the cyst is calcified rendering the serological diagnosis of intracranial hydatid disease a challenge. ${ }^{13}$

The differential diagnosis of intracranial cystic lesions is wide, including cystic and necrotic malignancies as well as a wide range of benign lesions such as abscesses. Differentiating these lesions is essential in the management and can be at times difficult. This is particularly important in cases of rare lesions, such as hydatid cysts. Therefore, imaging is essential in providing accurate diagnosis and guiding the management of this rare disease. CT and MRI demonstrate a well-defined spherical or oval homogeneous cystic lesion with a thin wall and smooth margins and imaging characteristics of the cystic component similar to that of CSF. ${ }^{1}$ On unenhanced CT, the cyst wall can be isodense or hyperdense when compared to the brain tissue. ${ }^{3}$ On MRI, the cyst wall usually has low signal intensity on both T1-weighted and T2-weighted images. ${ }^{3} 4$ Calcification of the wall is extremely rare, reported to be $<1 \%$ of all cases ${ }^{1} 34$ 14-16 and was noted in our case on pathological examination. Although the mass effect is prominent, surrounding oedema and rim enhancement are usually absent in untreated or uncomplicated cases, unlike what is typically seen in cerebral abscesses

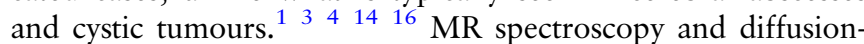
weighted imaging have recently been used to further aid in distinguishing brain hydatid cysts from cerebral abscesses and cystic or necrotic brain tumours. ${ }^{17-21}$ Kohli et al ${ }^{18}$ performed in vivo MR spectroscopy analysis in a patient of intracranial hydatid cyst and found an MR spectroscopy pattern different from that seen in other cystic lesions of the central nervous system. Besides lactate, alanine and acetate, there was a large resonance for pyruvate. ${ }^{18}$ Chand et al ${ }^{17}$ demonstrated mildly elevated choline, depressed creatine and NAA, and a large peak of lactate, pyruvate and acetate. ${ }^{17}$ Acetate and succinate are wellknown metabolic end products arising from microorganisms. ${ }^{22}$ Although succinate and acetate are not specific for hydatid cyst and are seen in abscesses and other parasitic diseases such as neurocysticercosis, ${ }^{23-25}$ pyruvate has been considered a specific in vivo marker for cestodal, in particular hydatid cysts. ${ }^{17-19}$

In our case of pathologically proven intracranial hydatid cyst, MR spectroscopy of the central cystic part of the lesion showed elevated choline as well as pyruvate and succinate picks. MR spectroscopy of the peripheral solid part of the lesion showed increased choline/NAA ratio and a large peak of lipids. As proton MR spectroscopy provides a non-invasive method for examining a wide variety of metabolites in the human brain, it can be used as an adjunctive modality to identify the nature of intracranial lesions including hydatid cysts.

Hydatid cysts have three layers; an outer pericyst which represents response of the host to the parasite, a middle laminated acellular membrane which permits the passage of nutrients and is impervious to bacteria, and an inner thin and translucent germinal layer. The infectious embryonic tapeworms (Scolices) develop from the brood capsule, which is an outpouching of the germinal layer. The thickness of the cysts layers depends on the hosting organ in which the cyst is located; these layers are often thick in hepatic hydatid cysts and sometimes visible in the brain.

The primary treatment of intracranial hydatid cysts is surgery and should be as radical as possible. Injection of formalin and evacuation of the cyst was the technique used until 1967.15 Thereafter, the Dowling technique has been used to remove the cyst as the most common and the gold standard method; unfortunately, some intraoperative difficulties may be encountered. Cyst rupture during surgery may occur in $28 \%$ of the cases. ${ }^{15} 26$ Cyst rupture into the subarachnoid space may lead to 
widespread dissemination followed by a severe anaphylactic response, in addition to a higher recurrence rate. In the case of intraoperative rupture, the operative site should be systematically and repeatedly cleaned with a solution of $3 \% \mathrm{NaCl}$ or $10 \%$ formaldehyde. Albendazole is recommended in cases of intraoperative rupture of the cysts and in case of recurrence. It is also recommended in inoperable cases of multifocal disease or involving vital brain structures. ${ }^{15}$ Preoperative albendazole therapy may effectively reduce the tension of an intracranial hydatid cyst. ${ }^{27} 28$ Indications for preoperative or postoperative therapy with albendazole or mebendazole include rupture, multiple organ involvement, multiple brain lesions, recurrence and preoperative volume reduction. ${ }^{29} 30$

\section{Conclusion}

Hydatid disease of the brain is a rare disease in non-endemic areas that should be included in the differential diagnosis of cystic lesions of the brain, especially in a child coming from a farming region. The diagnosis of the hydatid cyst could be very hard especially when serological tests are negative. It has a typical radiological appearance; however, CT scan and MRI are the most reliable means of preoperative diagnosis. Although CT is superior in detecting calcification of the cyst wall, when present, MRI is better in detecting multiplicity and defining the anatomic relationship of the lesion with the adjacent structures and helps in surgical planning. The MR spectroscopy findings of the present case were specific to what it may be present in cases of hydatid disease of the brain. In vivo MR spectroscopy may be used as an adjunct in the diagnosis of intracranial hydatid disease.

\section{Learning points}

- Eechinococcosis is a worldwide zoonosis produced by the larval stage of the Echinococcus tapeworm.

- Intracranial hydatid disease is a rare disease with a reported incidence of $1-2 \%$ of all cases.

- Most of the cysts are located in the supratentorial regions; most commonly in the parietal lobes.

- Cerebral hydatid cysts may not show any symptoms until they reach a considerably large size. Accurate preoperative diagnosis is challenging and imaging is essential to differentiate from other intracranial cystic lesions.

- MR spectroscopy is a novel imaging modality showing a specific pattern for hydatid cysts different from the other cystic lesions of brain. MR spectroscopy can be used as an important adjunct to imaging in the differential diagnosis of intracranial hydatids.

Competing interests None declared.

Patient consent Obtained.

Provenance and peer review Not commissioned; externally peer reviewed.

\section{REFERENCES}

1 Pedrosa I, Saíz A, Arrazola J, et al. Hydatid disease: radiologic and pathologic features and complications. Radiographics 2000;20:795-817.
2 Binesh F, Mehrabanian M, Navabii H. Primary brain hydatosis. BMJ Case Rep 2011;2011:bcr0620103099.

3 Bükte Y, Kemaloglu S, Nazaroglu H, et al. Cerebral hydatid disease: CT and MR imaging findings. Swiss Med Wkly 2004;134:459-67.

4 Polat P, Kantarci M, Alper F, et al. Hydatid disease from head to toe. Radiographics 2003;23:475-94.

5 Gupta S, Desai K, Goel A. Intracranial hydatid cyst: a report of five cases and review of literature. Neurol India 1999:47:214-17.

6 Demir K, Karsli AF, Kaya T, et al. Cerebral hydatid cysts: CT findings. Neuroradiology 1991;33:22-4.

7 Patrikar DM, Mitra KR, Bhutada VR. Cerebral hydatid disease. Australas Radiol 1993:37:226-7.

8 Vaquero J, Jiménez C, Martínez R. Growth of hydatid cysts evaluated by CT scanning after presumed cerebral hydatid embolism. Case report. J Neurosurg 1982; 57:837-8.

9 Nazaroğlu H, Ozateş M, Bilici A, et al. Multilocular cerebral hydatid disease with extracalvarial extension. AJR Am J Roentgenol 1999;172:1455-6.

10 Ciurea AV, Vasilescu G, Nuteanu L, et al. Cerebral hydatid cyst in children. Experience of 27 cases. Childs Nerv Syst 1995;11:679-85.

11 El-Shamam 0, Amer T, El-Atta MA. Magnetic resonance imaging of simple and infected hydatid cysts of the brain. Magn Reson Imaging 2001;19:965-74.

12 Singounas $E G$, Leventis $A S$, Sakas $D E$, et al. Successful treatment of intracerebral hydatid cysts with albendazole: case report and review of the literature. Neurosurgery 1992;31:571-4.

13 Eşgin $M$, Aktaş $M$, Coşkun S. [The investigation of antibody presence in the sera of patients with a suspicion of cystic echinococcosis by using indirect hemaglutination test (IHA)]. Turkiye Parazitol Derg 2007;31:283-7.

14 Beggs I. The radiology of hydatid disease. AJR Am J Roentgenol 1985; 145:639-48.

15 Najjar MW, Rajab Y, El-Beheiri Y. Intracranial hydatid cyst. Dilemma in diagnosis and management. Neurosciences (Riyadh) 2007;12:249-52.

16 Nurchi G, Floris F, Montaldo C, et al. Multiple cerebral hydatid disease: case report with magnetic resonance imaging study. Neurosurgery 1992;30:436-8.

17 Chand K, Kanodia AK, Manpreet G, et al. In vivo proton magnetic resonance spectroscopy in a known case of intracranial hydatid cyst. Neurol India 2005;53:337-8.

18 Kohli A, Gupta RK, Poptani H, et al. In vivo proton magnetic resonance spectroscopy in a case of intracranial hydatid cyst. Neurology 1995;45(Pt 1):562-4.

19 Jayakumar PN, Srikanth SG, Chandrashekar HS, et al. Pyruvate: an in vivo marker of cestodal infestation of the human brain on proton MR spectroscopy. J Magn Reson Imaging 2003;18:675-80.

20 Shukla-Dave A, Gupta RK, Roy R, et al. Prospective evaluation of in vivo proton MR spectroscopy in differentiation of similar appearing intracranial cystic lesions. Magn Reson Imaging 2001;19:103-10.

21 Yadav VK, Sudhakar SV, Panwar J. Pathognomonic MRI and MR spectroscopy findings in cerebral hydatid cyst. Acta Neurol Belg 2015.

22 Burtscher IM, Holtås S. In vivo proton MR spectroscopy of untreated and treated brain abscesses. AJNR Am J Neuroradiol 1999;20:1049-53.

23 Agarwal M, Chawla S, Husain N, et al. Higher succinate than acetate levels differentiate cerebral degenerating cysticerci from anaerobic abscesses on in-vivo proton MR spectroscopy. Neuroradiol 2004;46:211-15.

24 Mishra AM, Gupta RK, Jaggi RS, et al. Role of diffusion-weighted imaging and in vivo proton magnetic resonance spectroscopy in the differential diagnosis of ring-enhancing intracranial cystic mass lesions. J Comput Assist Tomogr 2004;28:540-7.

25 Dev R, Gupta RK, Poptani $\mathrm{H}$, et al. Role of in vivo proton magnetic resonance spectroscopy in the diagnosis and management of brain abscesses. Neurosurgery 1998;42:37-42.

26 Onal C, Unal F, Barlas O, et al. Long-term follow-up and results of thirty pediatric intracranial hydatid cysts: half a century of experience in the Department of Neurosurgery of the School of Medicine at the University of Istanbul (1952-2001). Pediatr Neurosurg 2001;35:72-81.

27 Altas $M$, Serarslan $Y$, Davran $R$, et al. The Dowling-Orlando technique in a giant primary cerebral hydatid cyst: a case report. Neurol Neurochir Pol 2010;44:304-7.

28 Todorov T, Vutova K, Mechkov G, et al. Chemotherapy of human cystic echinococcosis: comparative efficacy of mebendazole and albendazole. Ann Trop Med Parasitol 1992;86:59-66.

29 Bartosch C, Reis C, Castro L. Large solitary cerebral hydatid cyst. Arch Neurol 2011;68:946-7.

30 Nourbakhsh A, Vannemreddy P, Minagar A, et al. Hydatid disease of the central nervous system: a review of literature with an emphasis on Latin American countries. Neurol Res 2010;32:245-51. 
Copyright 2016 BMJ Publishing Group. All rights reserved. For permission to reuse any of this content visit http://group.bmj.com/group/rights-licensing/permissions.

BMJ Case Report Fellows may re-use this article for personal use and teaching without any further permission.

Become a Fellow of BMJ Case Reports today and you can:

- Submit as many cases as you like

- Enjoy fast sympathetic peer review and rapid publication of accepted articles

- Access all the published articles

- Re-use any of the published material for personal use and teaching without further permission

For information on Institutional Fellowships contact consortiasales@bmjgroup.com

Visit casereports.bmj.com for more articles like this and to become a Fellow 